\title{
The time of sentinel lymph node biopsy and neoadjuvant chemotherapy in clinical negative axilla breast cancer: before or after?
}

\author{
Zhao $\mathrm{Bi}^{1,2}$, Jingjing $\mathrm{Liu}^{3}$, Peng Chen², Xianrang Song ${ }^{2}$ \& Yongsheng Wang*,2 \\ ${ }^{1}$ School of Medicine \& Life Sciences, University of Jinan-Shandong Academy of Medical Sciences, Jinan, Shandong, PR China \\ ${ }^{2}$ Shandong Cancer Hospital and Institute, Shandong First Medical University and Shandong Academy of Medical Sciences, Jinan, \\ Shandong, PR China \\ ${ }^{3}$ Laboratory, Qingdao Municipal Hospital, Qingdao, Shandong, PR China \\ *Author for correspondence: wangysh2008@aliyun.com
}

\section{"the clinical nodal staging and molecular subtypes should be considered when choosing optimal time to perform SLNB following NAC."}

First draft submitted: 4 May 2019; Accepted for publication: 8 July 2019; Published online: 19 August 2019

Neoadjuvant chemotherapy (NAC) refers to systemic cytotoxic drug therapy for nondistant metastatic tumors before surgery, which is also known as preoperative chemotherapy or induction chemotherapy [1]. Pathologic complete response (pCR) is an independent prognostic factor, and several studies indicated that $\mathrm{pCR}$ was associated with molecular subtype [2-5]. In a woman who presented with a clinically node-negative $\left(\mathrm{cN}_{0}\right)$ disease and received NAC, sentinel lymph node biopsy (SLNB) is thought to be appropriate [6,7]. Before the start of AMAROS and ACSOG Z0011 trials, SLNB should be performed after NAC. But since the results of AMAROS and ACSOG Z0011 trials appeared, axillary lymph node dissection (ALND) could be avoided for patients with $\leq 2$ positive sentinel lymph nodes (SLNs) if they would receive axilla radiotherapy [8,9]. So, the optimal time of SLNB and NAC in patients with $\mathrm{cN}_{0}$ disease remains uncertain. For $\mathrm{cN}_{0}$ patients, 2017.V1/V2 NCCN breast cancer clinical practice guidelines recommend that it is feasible to perform SLNB before or after NAC, while 2018.V1/V2 NCCN guidelines have changed that SLNB preferably be performed after NAC [10,11]. In 2017, St Gallen Expert Consensus and conference, $95.7 \%$ of experts recommended SLNB for $\mathrm{cN}_{0}$ patients undergoing NAC. But the opinions were not consistent for the optimal time to perform SLNB and NAC. Sixty percent of experts supported SLNB should be performed after NAC, while $20 \%$ of experts believed that it was suitable for SLNB before NAC. The remaining experts supported that it is appropriate to perform SLNB before or after NAC [12,13].

The accurate assessment of the primary axillary tumor burden is the biggest advantage of the SLNB prior to NAC. It could accurately assess the primary tumor burden and help to guide the NAC regimen. At the same time, it is a gut feeling that performing an SLNB before the start of NAC for $\mathrm{CN}_{0}$ patients will reduce the morbidity more compared with an SLNB after NAC [14,15]. The 2019.V1 NCCN guide suggested that ALND will be a standard treatment for patients with any positive SLNs when they receive SLNB after NAC, including micro-metastasis and isolated tumor cells [13]. According to the results of the AMAROS and ACSOG Z0011 test, if axillary radiotherapy is accepted, ALND can be avoided for patients with less than two positive SLNs. The NSABP B-32 study and AMAROS trial found that about $30 \%$ of $\mathrm{cN}_{0}$ patients undergoing SLNB before NAC were SLNs positive [8,16]. ACOSOG Z0011 trial showed that among patients with cT1-2N0 breast cancer undergoing upfront BCS and planned whole breast radiation therapy, ALND was warranted for three or more positive SLNs. According to the ASCOG Z0011 trial and the AMAROS trial, about $80 \%$ of $\mathrm{cN}_{0}$ patients (SLNs positive) were those of one to two SLNs positive who were eligible for receiving whole breast radiotherapy or axillary radiotherapy after mastectomy to avoid ALND, which made about $94 \%$ (30\% multiplied by $80 \%$ plus $70 \%$ was $94 \%$ ) of patients avoid ALND.

Based on the application of ACOSOG Z0011 trial criteria, Pilewskie [17] made a retrospective study and result showed that receipt of NAC compared with upfront BCS remained significantly associated with higher odds 
of ALND in the hormone receptor positive/HER-2 negative (HR+/HER2-) subtype (hazard ratio [HR]: 3.35; $\mathrm{p}<0.001$ ), whereas NAC versus upfront mastectomy remained significantly associated with lower odds of ALND in the HER2+ and TN subtypes (HR: 0.19, $\mathrm{p}<0.001$; HR: 0.25, $\mathrm{p}=0.007$, respectively) [13]. And in our study, we thought that combined with the results of AMAROS and ASCOG Z0011 trial, the rate of avoiding ALND when performing SLNB after NAC was associated with the axillary pCR (apCR) rate after NAC. Our recent study showed that in patients with $\mathrm{cN}_{0}$ disease, the rate of pathological lymph node positive $\left(\mathrm{ypN}_{+}\right)$after NAC was $19.4 \%$ (13/67), and it was 28.1, 13.3 and 10.0\%, respectively, among HR+/HER2-, HER2+ and TN patients [13]. The rates of $\mathrm{ypN}_{0}$ after NAC in patients with HER2+ and TN were $90.0 \%(18 / 20)$ and $86.7 \%$ (13/15), respectively, which were significantly higher than HR+/HER2- patients $(71.8 \%, 23 / 32)$.

Since patients with $\mathrm{cN}_{0}$ disease were unable to assess axillary status effectively and the sample size was small, we predicted the apCR of $\mathrm{cN}_{0}$ patients after $\mathrm{NAC}$ by evaluating the apCR of patients with clinical node positive $\left(\mathrm{cN}_{+}\right)$ disease [13]. Gentile et al. found that the rates of apCR after NAC in $\mathrm{cN}_{+}$patients with HER2+ (with targeted therapy) and TN were 63.0 and $41.0 \%$, respectively, which were significantly higher than that of HR+/HER2patients $(17.0 \%, \mathrm{p}<0.001)$. At the same time, we also found apCR after NAC was significantly associated with molecular subtypes. The apCR rate was 21.2, 53.6 and 58.2\%, respectively among patients with HR+/HER2-, TN and HER2+ subtypes. Based on the AMAROS and ASCOG Z0011 trial, there will be $94 \%$ of $\mathrm{cN}_{0}$ patients who could avoid ALND (30\% multiplied by $80 \%$ plus $70 \%$ was $94 \%$ ) if performing SLNB before NAC [6]. For $\mathrm{cN}_{0}$ patients with HR+/HER2- subtype, if performed SLNB after NAC, there will be $76.4 \%$ of patients could avoid ALND (30\% multiplied by $21.2 \%$ plus $70 \%$ was $76.4 \%$ ). On the contrary, the apCR was high in TN and HER2 + patients with $\mathrm{cN}_{0}$ disease after NAC; using the same method, those patients might have more chance (30\% multiplied by $53.6 \%$ plus $70 \%$ was $86.1 \%$, and $30 \%$ multiplied by $58.2 \%$ plus $70 \%$ was $87.5 \%$, respectively) to avoid ALND compared with HR+/HER2- patients.

Although TN (94 vs $86.1 \%$ ) and HER2+ (94 vs 87.5\%) patients performing SLNB prior to NAC had more chance to avoid ALND compared with SLNB after NAC, NAC had another advantage that it could offer an opportunity to evaluate the efficacy of chemotherapy on primary tumor in vivo. Furthermore, the residual tumor burden after NAC could assess prognosis of patients and guide future treatment, and it was a more meaningful predictor of localregional recurrence than before NAC. Although there were $7-8 \%$ of TN and HER2+ patients could benefit from avoiding ALND when performed SLNB prior to NAC, the total pCR after NAC could have more pronounced effect on prognosis among these patients. The total pCR in TN and HER2+ patients after NAC could also be used as a surrogate marker for long-term survival to better assess prognosis, whereas the total pCR in HR+/HER2- patients have little thing to do with long-term survival benefit.

At the same time, our result showed that $\mathrm{ypN}_{0}$ after $\mathrm{NAC}$ was significantly correlated with molecular subtypes of the breast tumor in initial $\mathrm{cN}_{0}$ patients $(\mathrm{p}<0.001)$. The rate of $\mathrm{ypN}_{0}$ after $\mathrm{NAC}$ in $\mathrm{cN}_{0}$ patients was $80.6 \%$, especially in patients with HER2+ (90.0\%) and TN (86.7\%), making it possible to selectively avoid axillary surgery after NAC, which would contribute to reduce postoperative complications, improve patient quality of life and reduce medical costs.

In conclusion, the clinical nodal staging and molecular subtypes should be considered when choosing optimal time to perform SLNB following NAC. Combining the apCR in different molecular subtypes of $\mathrm{cN}_{+}$patients and excellent loco-regional control of AOSOG Z0011 and AMAROS trials in $\mathrm{cN}_{0}$ patients, it would be preferable to perform SLNB prior to NAC to reduce the risk of ALND for $\mathrm{cN}_{0}$ patients with $\mathrm{HR}+/ \mathrm{HER} 2-$ - SLNB after NAC for $\mathrm{cN}_{0}$ patients with HER2+ and TN could better avoid ALND. In view of the high ypN $\mathrm{N}_{0}$ rate after NAC in $\mathrm{cN}_{0}$ patients, axillary surgical staging might be selectively eliminated, especially in patients with HER2+ and TN breast cancer.

\section{Financial \& competing interests disclosure}

The authors have no relevant affiliations or financial involvement with any organization or entity with a financial interest in or financial conflict with the subject matter or materials discussed in the manuscript. This includes employment, consultancies, honoraria, stock ownership or options, expert testimony, grants or patents received or pending, or royalties.

No writing assistance was utilized in the production of this manuscript. 


\section{References}

1. Fisher B, Brown A, Mamounas $\mathrm{E}$ et al. Effect of preoperative chemotherapy on local regional disease in women with operable breast cancer: findings from National Surgical Adjuvant Breast and Bowel Project B-18. J. Clin. Oncol. 15, 2483-2493 (1997).

2. Lori F, George P, Emily C et al. Tumor biology predicts pathologic complete response to neoadjuvant chemotherapy in patients presenting with locally advanced breast cancer. Ann. Surg. Oncol. 34(13), 3896-3902 (2017).

3. Boughey JC, McCall LM, Ballman KV et al. Tumor biology correlates with rates of breast-conserving surgery and pathologic complete response after neoadjuvant chemotherapy for breast cancer: findings from the ACOSOG Z1071 (Alliance) prospective multicenter clinical trial. Ann. Surg. 260(4), 608-616 (2014).

4. Boileau JF, Poirier B, Basik M et al. Sentinel node biopsy after neoadjuvant chemotherapy in biopsy-proven node-positive breast cancer: the SNFNAC study. J. Clin. Oncol. 33(3), 258-264 (2015).

5. Mamtani A, Andrea V, Tari A et al. How often does neoadjuvant chemotherapy avoid axillary dissection in patients with histologically confirmed nodal metastases? Results of a prospective study. Ann. Surg. Oncol. 23(11), 3467-3474 (2016).

6. Kumar A, Puri R, Gadgil PV et al. Sentinel lymph node biopsy in primary breast cancer: window to management of the axilla. World J. Surg. 36(7), 1453-1459 (2012).

7. Diego E, McAuliffe P, Soran A et al. Axillary staging after neoadjuvant chemotherapy for breast cancer: a pilot study combining sentinel lymph node biopsy with radioactive seed localization of pretreatment positive axillary lymph nodes. Ann. Surg. Oncol. 23(5), 1549-1553 (2016).

8. Mila D, Geertjan T, Marieke ES et al. Radiotherapy or surgery of the axilla after a positive sentinel node in breast cancer (EORTC 10981-22023 AMAROS): a random, multi-center, open-label, Phase III non-inferiority trial. Lancet Oncol. 15(12), 1303-1310 (2014).

9. Giuliano AE, Hunt KK, Ballman KV et al. Axillary dissection vs no axillary dissection in women with invasive breast cancer and sentinel node metastasis: a randomized clinical trial. JAMA 305, 569-575 (2011).

10. Grasishar WJ, Anderson BO, Balassanian R et al. Breast Cancer, Version 3. 2017 Featured Updates to the NCCN Guidelines [EB]. National Comprehensive Cancer Network. www.NCCN.org

11. Grasishar WJ, Anderson BO, Abraham J et al. Breast Cancer, Version 2. 2018 Featured Updates to the NCCN Guidelines [EB]. National Comprehensive Cancer Network. www.NCCN.org

12. Curigliano G, Burstein H, Winer E et al. De-escalating and escalating treatments for early-stage breast cancer: the St. Gallen International Expert Consensus Conference on the Primary Therapy of Early Breast Cancer. Ann. Oncol. 28, 1700-1712 (2017).

13. Bi Z, Liu JJ, Wang YS et al. Neoadjuvant chemotherapy and timing of sentinel lymph node biopsy in different molecular subtypes of breast cancer with clinically negative axilla. Breast Cancer 26(3), 373-377 (2019).

14. Audree B, Wei T, Savitri K et al. Identification of patients with documented pathologic complete response in the breast after neoadjuvant chemotherapy for omission of axillary surgery. JAMA 152(7), 665-670 (2017).

15. Giuliano AE, Hunt KK, Ballman KV et al. Axillary dissection vs no axillary dissection in women with invasive breast cancer and sentinel node metastasis: a randomized clinical trial. JAMA 305, 569-575 (2011).

16. Krag DN, Anderson SJ, Julian TB et al. Sentinel-lymph-node resection compared with conventional axillary-lymph-node dissection in clinically node-negative patients with breast cancer: overall survival findings from the NSABP B-32 random Phase III trial. Lancet Oncol. 11(10), 908-909 (2010).

17. Pilewskie M, Zabor E, Mamtani A et al. The optimal treatment plan to avoid axillary lymph node dissection in early-stage breast cancer patients differs by surgical strategy and tumor subtype. Ann. Surg. Oncol. 24(12), 3527-3533 (2017). 
